dichte Bekleidung, bestehend aus unterseits schwärzlichen Drüsenhaaren. Die Bekleidung der Hüllblättchen besteht aus Sternhaaren, sowie aus sehr kurzen, schwärzlichen einfachen und Drüsenhaaren, welch letztere besonders reichlich aufgetragen sind.

Standort: Auf sonnigen Grastriften zwischen Bodnarówka und Zubrza bei Lemberg zahlreich. Dr. WoZoszezak sammelte dieselbe Pflanze in der unteren Region der Kołomyjaer Karpathen.

Anmerkung: Systematisch am nächsten verwandt ist mein $\boldsymbol{H}$. Andraejowskii mit dem ostkarpathischen $H$. roxolanicum Rehmann; dieses letztere unterscheidet sich jedoch auf den ersten Blick von H. Andrzejowskii durch orangengelbe Blüthen. Sehr nahe Verwandtschaft weist $\boldsymbol{H}$. Andrzejowskii auch mit $\boldsymbol{H}$. Bubelae mihi von Wsetin in Mähren auf, welches Herr J. Bubela in den letzten Jahren in der "Linnaea" unter dem Namen $\boldsymbol{H}$. glomeratum Froehl. ausgegeben hat, jedoch weicht mein $\boldsymbol{H}$. Andrrejowskii von demselben durch fast zweimal kleinere Köpfchen und andere Gestalt der Grundblätter ab.

Lemberg, im April 1888.

\title{
Zur Frage der Einschleppung und Verwilderung von Pflanzen.
}

\section{Von Prof. A. Zimmeter.}

Herr Dr. Josef Murr hat im "Botanischen Centralblatt" 1888, Nr. 4 bis 7 einen 15 Seiten umfassenden Artikel: "Ueber die Einschleppung und Verwilderung von Pflanzenarten im mittleren Nordtirol" veröffentlicht.

Dor Verfasser hat in dieser Schrift seine schon in der Oester. bot. Zeitschr. in den Jahren 1881 bis 1884 publicirten Beobachtungen zu einem harmonischen Ganzen zusammengefasst und durch zahlreich $\theta$ neue Beobachtungen vermehrt, sowio auch interessantero Funde anderer Botaniker, wie Graf Sarnthein, v. Benz, Dalla Torre, Evers, Hellweger, Gremblich etc. namhaft gemacht.

Es ist eine sehr verdienstliche Aufgabe, derlei oft in den verschiedensten Zeitschriften zerstreute Notizen zusammenzufassen, da eine derartige Zusammenstellung nicht nur für den Pflanzen-Geographen, sondern insbesondere auch für Denjenigen werthvoll ist, der sich in der betreffenden Gegend orientiren will; sie hat Werth für den Studierenden, sowie auch für den in der bezüglichen Gegend aufgewachsenen Botaniker, der nur zu oft versucht ist, gerade jene Pflanzen, die er von seiner Jugend an in seiner Heimat gefunden, für wirklich einheimische Gewächse zu halten, während doch ein gut Theil davon seit mehr oder weniger langer Zeit eingewandert war. 
Von hohem Interesse wäre es dabei allerdings, auch den Zeitpunkt der Einwanderung, wenn auch nur annäherungsweise festzusetzen, zu welchem Zwecke eben einmal der Anfang gemacht werden muss. Dass bei derlei höchst difficilen Versuchen Irrungen unvermeidlich sind und erst im Laufe der Zeit geklärt werden könnon, versteht sich wohl von selbst; es müssen dabei eben nicht nur die betreffenden Arten auf botanischen Ausflügen notirt werden, man darf sich nicht nur auf die eigene Erinnerung verlassen, sondern muss zu dem Behufe die vorhandene Literatur fleissig benützen und auch, wo möglich, ältere Herbarien. Leider findet sich in diesen oft genug der Standort einer Pflanze nicht mit der wünschenswerthen Exactheit angegeben, um ein allenfallsiges Wiederauffinden der fraglichen Pflanze zu ermöglichen.

Murr's Arbeit zeigt von fleissigem Durchstöbern der Gegend, er hat gewissenhaft die Funde seiner botanischen. Freunde neuerer Zeit benützt, or hat auch die Literatur nach Thunlichkeit zu Rathe gezogen, leider jedoch weniger Gewicht auf ältere Herbare gelegt. Ich will nun in Folgendem gewiss nicht der - ich wiederhole es sehr verdienstlichen Arbeit Murr's nahe treten, sondern möchte nur auf einige Punkte aufmerksam machen, die mir bei der Durchsicht obgenannter Schrift aufgefallen sind.

Vor Allem legt, wie mir scheint, der Verfasser ein viel zu hohes Gewicht auf die Bodeutung des Scirocco für den Transport. von Samen und Früchten, und ich möchte in dieser Beziehung auf den hochinteressanten Aufsatz Kerner's aufmerksam machen, der dieses Thema in der Zeitschrift des dentschen und österreichisehen Alpenvereins 1871, pag. 144, behandelt. Es ist mir nicht gut denkbar, wie die ziemlich schweren Früchte, respective Samen von Ostrya carpinifolia, Dorycnium decumbens, Ranunculus parnassifolius, Braya alpina, Malva alcea u. a. dureh mehr oder minder hochgehende Luftströme so weite Strecken hindurch transportirt werden sollten, ohne dass - selbst wenn wir eine schrittweise Verbreitung annehmen wollen - in jenen Gegenden, die zwischen den nordtirolischen Standorten und ihrer südlicheren Heimat liegen, die Pflanzen an den gewiss vorhandenen passenden Localitäten auftreten sollten. Manche davon dürften vielleicht aber als Relicte einer früheren Zeit anzusehen sein, andere sind vielleicht zufällig eingeschleppt worden.

Auch die Zeit der "Einschleppung" mancher Arten dürfte Murr zu spät angesetzt haben; so liegen mir beispielsweise eine Reihe von Pflanzen in meinem Herbare aus meines Vaters Hand vor, die derselbe in den Fünfziger-Jahren oder Anfangs der SechzigerJahre sammelte; so die Ostrya, die damals schon blühte, also ein kräftiges Bäumchen war; Euphorbia exigua fand mein Vator auf Ruderal- nicht Ackerboden bei der Schweinsbrücke, Cuccubalus baccifer beim grossen Ziegelstadel in der Haller Au, Reseda luteola beim Weiler Kranebitten, Potentilla supina bei Mühlau etc.; letztere Pflanze ist gewiss kein Gartenflüchtling, da sie kaum Jemand als Zierpflanze bauen dürfte. 
Ich lege jedoch auf die zuletzt berührte, ohnedies geringfügige Zoitdifferenz um so weniger Werth, als eben Herr Murr von den Funden moines Vaters nichts wissen konnte, da derselbe in der letzten Zeit nichts mehr publicirte.

Dagegen möchte ich an dieser Stelle die Aufmerksamkeit alDerer, die sich mit ähnlichen Zusammenstellungen befassen, auf die Berücksichtigung älterer Herbare lenken. Für die Gegend von Innsl bruck hat seinerzeit Kerner in der Oesterr. bot. Zeitschrift 1866, XVI., p. 137 ff. auf das alte, gegenwärtig im Besitze des Ferdinandeums in Innsbruck befindliche Herbar Hypolit v. Guarinoni's anfmerksam gemacht, das derselbe wahrscheinlich zwischen 1610 und 1630 angelegt haben dürfte. ${ }^{1}$ ) Es enthält circa 600 Arten, deren heutige Nomenclatur Kerner 1. c. aufführt. Kerner zeigt, dass Guarinoni wohl alle Pflanzen aus der Umgebung von Innsbruck und Hall requirirt hat; die Zahl der zuverlässig aus Gärten stammenden Pflanzen beträgt 40. Aus der Durchsicht des Herbars ergibt sich nach Kerner:

1. Dass die einzelnen Arten sich während dritthalb Jahrhunderten nicht im geringsten geändert haben.

2. Dass gewisse jetzt in der Umgebung Innsbrucks seltenere Pflanzen, die dermalen oft nur an einem einzigen versteckten Standorte vorkommen, wie z. B. Iris sibirica, Asperugo procumbens, Malaxis monophyllus damals häufiger gewesen sein müssen.

3. Dass eine Reihe von Pflanzen aus der Flora von Innsbruck soit dieser Zeit verschwunden sein müsse, wohin jedoch nur: Linnaea borealis, Scolopendrium officinarum und Scandix Pecten Veneris gehören, von denen letztere offenbar mit dem Getreide eingeschleppt wurde und wieder verschwand, während die Standorte der beiden erstgenannten mit der Reducirung der Wälder aus der nächsten Umgebung verschwanden.

4. Dass von Ackerunkräutern und Ruderalpflanzen die meisten schon damals vorhanden waren, wie z. B. Alchimilla arvensis, Lycopsis arvensis, Patentilla supina, Chenopodium murale, hybridum, Vulvaria und Asperugo procumbens, alles Pflanzen, die gewiss in unserer Gegend nicht ursprünglich heimisch sind, sondern wohl schon lange vor Guarinoni eingewandert waren und nicht erst in letater Zeit. ${ }^{2}$ )

1) Ueber altere Herbare vergleiche man Kreutzer: „Das Herbar“ Wien 1864; das älteste Herbar dürfte nach J. Münter (Oesterr. bot. Zeitschr. 1866, p. 201) das Rauwolff'sche Herbar aus der Zeit von 1560-1563 im Leydener Museum sein; das älteste auf uns gekommene Herbar in Oesterreich dürfte nach Kreutzer's Schrift aus der Mitte des 17. Jahrhunderts von einem gewissen Angermann stammen und befand sich 1864 noch im Besitze der Familie Peer in Brixen.

$\left.{ }^{2}\right)$ Einige bei Guarinoni vorkommende Pflanzen wären noch: Agrostemma Githago, Alyssum calyeinum, Bryonia alba, Delphinium Consolida, Dorycnium decumbens, Lamium amplexicaule, Lepidium ruderale, Saponaria ocymoides, Veronica triphyllos etc. 
Von jenen Pflanzen nun, welche, wenn wir obige Darstellung Kerner's, die ich nur auszugs- und probeweise mir hier wiederzugeben und in Erinnerung zu bringen erlaubte, berücksichtigen, wirklich erst seit neuerer Zeit, etwa seit der Zeit des Bahnbaues in Tirol (1858), in Innsbrucks Umgebung eingewandert, respective verwildert sein dürften, erlaube ich mir aus Murr's Arbeit nur einige der interessanteren hier kurz anzuführen, indem ich alle Jene, die sich hiefür näher interessiren, auf die Arbeit selbst vorwejse. Es finden sich unter vielen anderen: Stenactis bellidiflora L., Centaurea nigrescens W., Silene Anglica L., Sinapis dissecta Lag., Linum perenne L., Brassica nigra Koch, Vaccaria pyramidata Fl. d. W., Ambrosia artemisiaefolia L., Setaria italica Beauv, Centaurea Biebersteinii Cand., Salvia sylvestris L. (von Kravogl 1871, nicht von mir entdeckt) etc.

Innsbruck, im März 1888.

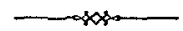

\section{Geum spurium C. A. Mey. in Ungarn und G. montanum var. geminiflorum $\mathrm{m}$.}

Von Dr. Vincenz v. Borbás.

Es ist wohl bekannt, dass die Blumenblätter des ziemlich verbreiteten Geum urbanum verkehrt eiförmig, klein und kürzer sind, als der Kelch. ${ }^{1}$ ) In Gussone's Fl. Sic. I (1842) p. 575 finden wir aber eine var. australe Guss. dieser Art beschrieben, "floribus erectis, calycibus petala rotundata aequantibus". Die Beschreibung der vegetativen Theile citire ich hier nicht, denn sie scheinen von jenen des typischen $G$. urbanum nicht erheblich abzuweichen. In Plant. vasc. in insula Inarime p. 118 werden von Gussone die "petala rotundata abrupte in unguem brevissimis attenuata" wiederum erwähnt und noch, was von Scheutz in seinem Prodromus Monogr. Georum p. 24 nicht reproducirt ist, "aristae purpurascentes incurvae, appendicibus angulo recto ab arista inflexis. Habitus a speciei typo nonnihil alienus. Geum intermedium Koch omnino diversum" beigetragen.

Die Petala des var. australe nähern sich also jenen des $G$. aleppicum Jcq., und habe ich der Beschreibung Gussone's entsprechende Formen bei Fiume, Ogulin und Adelsberg in Krain gesammelt, Exemplare, an welchen die Petala länger sind als der Kelch.

Wenn man aber in Siebonbürgen von dem Bade des Büdösberges, wo auch Hypericum umbellatum Kern. (Bot. Centralbl. VIII. pag. 391) und Hieracium sulphureum Döll. etc. wächst, zu dem

1) Kittel, Taschenbuch der Flora DeutschI. II. p. 1075. 